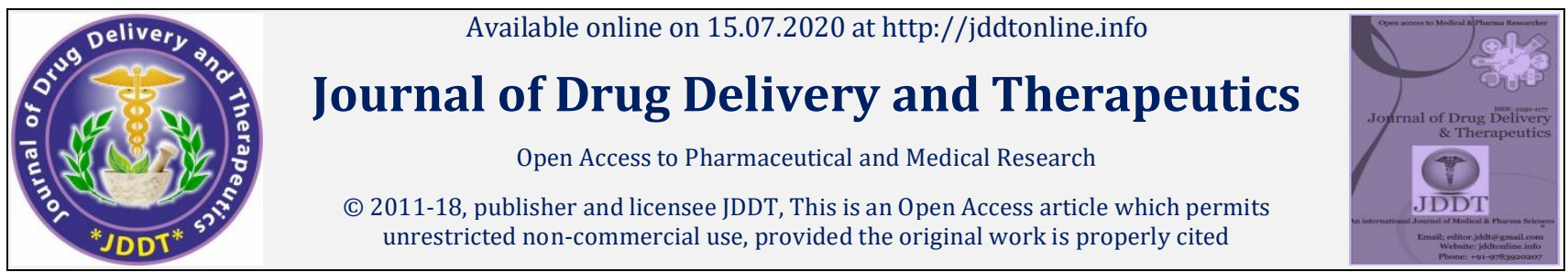

Open $\odot$ Access

Research Article

\title{
Designing, Cytotoxic Evaluation, Molecular Docking and in Silico Pharmacokinetic Prediction of New Hydrocortisone Derivatives as Anti- Asthmatics Drugs
}

\author{
Walid Bououden*, Yacine Benguerba \\ Laboratoire des Matériaux Polymères Multiphasiques, LMPMP, Université Ferhat ABBAS Sétif-1, Sétif 19000, Algeria
}

\begin{abstract}
A series of new 20 corticosteroids were subjected to molecular property prediction. The Molecular, Physicochemical, and Biolo gical properties were determined using Molinspiration Cheminformatics software. These compounds were further subjected to Toxicity Predictions using the Osiris Software. The calculated drug-related properties of the designed molecules were similar to those found in most marketed drugs. Amongst the proposed molecules, fourteen promising candidates can be considered as promising structures for the synthesis of new and more effective anti-asthmatic drugs. Result indicates that the derivatives are orally active molecules. In-silico ADME and toxicity prediction was accomplished with the help of Swiss-ADMET tool provides the latest and most inclusive for diverse chemicals associated with known Absorption, Distribution, Metabolism, Excretion and Toxicity profiles. furthermore, BBB (Blood brain barrier) penetration, HIA (Human intestinal absorption), Caco-2 cell permeability and Ames test were calculated using ADMET web-based query tools incorporating a molecular build in interface enable the database to be queried by Smiles and structural similarity search. According to molecular docking results, derivatives No 4, 10 and 11 showed better docking Scores values compared to other derivatives and also dexamethasone and hydrocortisone.
\end{abstract}

Keywords: Corticosteroids, Drug-likeness, Lipophilicity, Anti-asthmatic, ADME.

Article Info: Received 12 April 2020; Review Completed 14 June 2020; Accepted 21 June 2020; Available online 15 July 2020

\section{Cite this article as:}

Bououden W, Benguerba Y, Designing, Cytotoxic Evaluation, Molecular Docking and in Silico Pharmacokinetic Prediction of New Hydrocortisone Derivatives as Anti-Asthmatics Drugs, Journal of Drug Delivery and Therapeutics. 2020; 10(4):8-16 http://dx.doi.org/10.22270/jddt.v10i4.4128

*Address for Correspondence:

Walid Bououden, Laboratoire des Matériaux Polymères Multiphasiques, LMPMP, Université Ferhat ABBAS Sétif-1, Sétif 19000, Algeria

\section{INTRODUCTION}

Systemic oral corticosteroids (OCS) represent a class of drugs often used in the management of severe asthma. Despite their recognized safety risks, the updated report from the Global Asthma Initiative [1] recommends shortterm low-dose OCS for the management of exacerbations and as a supplement to maintenance therapy with corticosteroids inhaled (ICS) plus long-acting $\beta 2$ agonists (LABA)) in severe asthma for better disease control [2]. However, only empirical evidence appears to support the effectiveness of OCS in reducing exacerbation. In addition, a recent 20 -year observational study in severe asthma patients showed that those who were addicted to OCS had high mortality $50 \%$ of patients died) and had high exacerbation rates [3]. Emerging biological agents targeting molecular pathways of asthma have shown great promise in reducing the need for OCS [4]. Omalizumab, targeting immunoglobulins E (IgE), reduces exacerbations in patients with severe allergic asthma, regardless of the number of blood eosinophils [5]. Other biologics targeting specific interleukins (IL), such as anti-IL5 (mepolizumab and reslizumab), anti-IL5 R $\alpha$ (benralizumab) and anti IL4 R $\alpha$ (dupilumab) also reduce exacerbations in patients with severe eosinophilic asthma, without the main safety concerns that overshadow the OCS. As a first-line

ISSN: 2250-1177 treatment in severe asthma, GINA recommends adding these biological agents and / or long-acting muscarinic antagonists (LAMA) to an ICS and LABA combination [1], Inhaled corticosteroids (ISC ) are still the first-line anti-inflammatory therapy for persistent asthma of all severities [6]. In fact, they reduce the inflammation of the respiratory tract and, therefore, influence hyperresponsiveness. In this way, CSIs improve lung function and the severity of symptoms [7]. They are also effective in preventing or reducing the rate of asthma exacerbations. The anti-inflammatory activity of ICS is linked to their ability to target all the cells involved in asthmatic inflammation [8]. There is strong evidence that ICS suppress the expression and release of a large amount of inflammatory mediators and growth factors for epithelial cells in the primary respiratory tract, possibly via targeting nuclear factor or activator protein 1 (AP- 1) and by modulating the acetylation / deacetylation of histones [9]

Corticosteroid receptors, including GR and MR, are widely expressed in the body and are implicated in many diseases and health outcomes. GR and MR mediate the actions of glucocorticoids and mineralocorticoids, respectively belonging to a family of steroids involved in many physiological processes. Disturbances in the action of glucocorticoids have been linked to birth defects, mood and 
cognitive disorders, cancer, immune diseases, allergic diseases, metabolic dysfunctions, and cardiovascular diseases [10].

Several ICSs have been approved for the treatment of asthma. They include beclomethasone dipropionate, budesonide, ciclesonide, flunisolide, fluticasone furoate, fluticasone propionate, mometasone furoate and triamcinolone acetate. The profile of each ICS is critically influenced by the difference in their characteristics [11], but also, it is important to note that the significant differences in the selectivity, potency and physicochemical properties of glucocorticoid receptors (GR) are essential for defining the PD profile of an ICS [12].

The aim of this study is to create a novel inhibitor in the treatment of asthma disease based on the understanding of the structure-activity relationship carried out in a previous study [13]. To release this work, Molinspiration Cheminformatics, Osiris Software, Swiss-ADMET and (Schrodinger, Maestro) software were used.

\section{COMPUTATIONAL STUDIES}

For predicting the pharmacokinetic and drug-likeness properties of the major compounds of Hydrocortisone derivatives, the following online platforms were used:
SwissADME, Molinspiration and Osiris Data Warrior. SwissADME enables predictions for passive human gastrointestinal absorption (HIA) and blood-brain barrier (BBB) permeation of compounds, as well as the assessment of whether a compound is a substrate of P-gp and an inhibitor of CYP isoenzyme family. The knowledge about compounds being substrates or non-substrates of P-gp is essential for the evaluation of active efflux through biological membranes. The risks of toxicity (mutagenicity, tumorigenicity, irritability and reproductive effects) and the physicochemical properties (drug similarity and drug score) for compounds 1-20 were calculated by the methodology developed by Osiris. Various types of toxicities including oncogenicity, neurotoxicity, teratogenicity, immunotoxicity, etc. were generated and toxicity profile of molecule noted. Molinspiration Cheminformatics offers broad range of tools supporting molecule manipulation and processing, including SMILES and SDfile conversion by giving information about physicochemical descriptors and bioactivity relevant properties. All the parameters for drug-likeness were predicted and calculated according to the Lipinski's rule-offive. Moreover, to better understand the affinity between the drug and the receptor, molecular docking was carried out using Maestro version 11.1 Software.

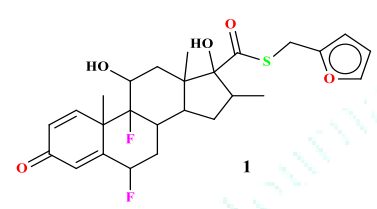

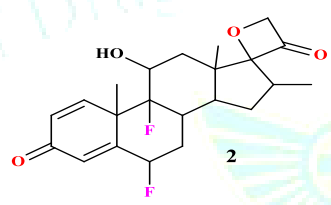

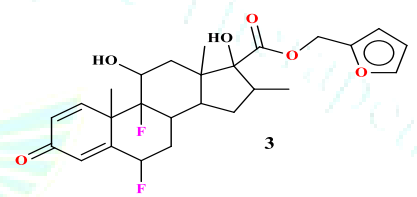

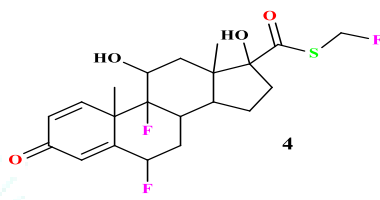

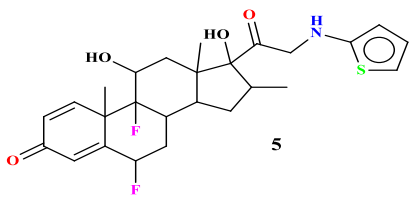<smiles>CC1=CC(=O)C=CC12CC(C)CC1C3CCC4(OCC4=O)C3(C)CC(C)[C@]12C</smiles>

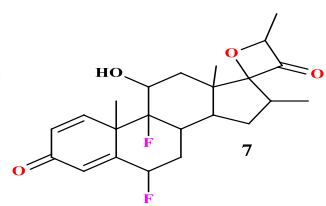<smiles></smiles>

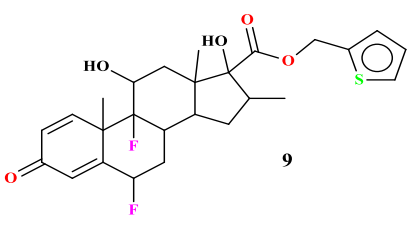<smiles>C/C=C/NC(=O)[C@@]1(C)C(C)CC2C3CC(C)C4=CC(=O)C=CC4(C)C3(C)C[C@H](C)CC21C</smiles>

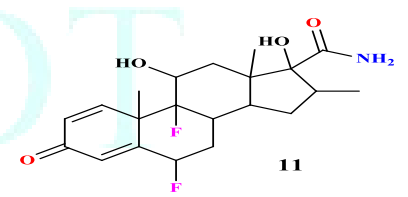

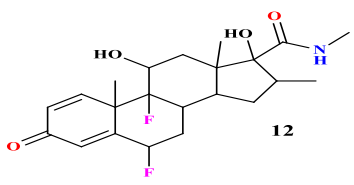<smiles>Cc1ccc(NCC(=O)C2C(C)CC3C4CCC5=CC(=O)C=CC5(C)C4(C)C(C)CC(C)C32C)s1</smiles>

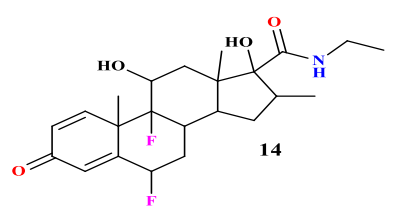<smiles>Cc1ccc(CSCC(=O)C2(C)C(C)CC3C4CCC5=CC(=O)C=CC5C4C(O)C(O)CC32C)s1</smiles>

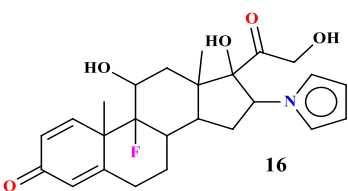<smiles>C=C(NOC)C(=O)C1(C)C(C)CC2C3CC(F)C4=CC(=O)C=CC4(C)C3(C)C(O)CC21C</smiles><smiles>Cc1ccc(CCC(=O)[C@]2(C)C[C@H](C)CC3(C)C(CC2C)C2CC(C)C4=CC(=O)C=CC4(C)C23)o1</smiles><smiles></smiles>

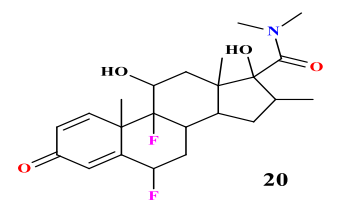<smiles>CC1CC2C3CCC4=CC(=O)C=CC4(C)C3(C)C(O)CC2(C)C1(C)C(=O)CO</smiles>

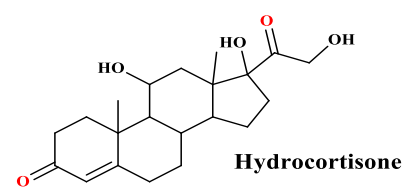

Figure 1: Molecular structure of the compounds (1-20) and standard drugs. 


\section{RESULTS AND DISCUSSION}

\section{Toxicity prediction}

The toxicity risk predictor locates fragments that indicate a potential risk of toxicity in a molecule [14]. Toxicity risk alerts indicate that the drawn structure may be dangerous for the specified risk category. From the data presented in the Table 1. , it is obvious that the analyzed compounds are supposed to be non-mutagenic, non-tumorigenic, nonirritant and without effects on reproduction with the exception of molecule 2, 3, 8, 12, 15 and 18 which is at low risk compared to the natural hormone.

The aqueous solubility of a compound significantly affects its absorption and distribution characteristics. Typically, low solubility is accompanied by poor absorption and, therefore, the general objective is to avoid poorly soluble compounds. Our estimated $(\log S$ ) value is a basic (stripped) logarithm of the solubility of a compound. It should be noted that more than $80 \%$ of the drugs on the market have an (estimated) log value greater than -4 . In the case of compounds 1-20, 9 elements have an acceptable aqueous solubility while a single compound is very soluble.
Drug resemblance is defined as a complex balance between various molecular properties and structural characteristics showing whether a molecule is like known drugs [15]. it should be mentioned that a positive value indicates that the designed molecule contains fragments which are frequently present in commercial drugs.

The data obtained Table 1. revealed that the analyzed compounds have positive drug similarity values and therefore there is a presence of the fragments in the commercial drugs. In contrast, 13 items in the set have higher drug similarity values than the standard drug, namely: dexamethasone.

The drug score combines drug similarity, miLogP, solubility, molecular weight and toxicity risks into a practical value that can be used to judge the overall potential of the compound to qualify for a drug [16]. indeed, a value of 0.5 or more makes the compound a promising advance for the development of future safe and effective drugs. in addition, three compounds have been found to be less than the typical value, but this does not indicate that these drugs are not effective compared to the natural hormone (hydrocortisone). The overall drug score values for most of the compounds have good drug score values.

Table 1: Toxicity risks, drug-likeness, and drug score of compounds (1-20) and standard drugs

\begin{tabular}{|c|c|c|c|c|c|c|c|}
\hline \multirow[b]{2}{*}{ Comp. $\mathrm{N}^{\circ}$} & \multicolumn{4}{|c|}{ Toxicity Risks } & \multirow[b]{2}{*}{ Solubility } & \multirow{2}{*}{$\begin{array}{c}\text { Drug- } \\
\text { likeness }\end{array}$} & \multirow{2}{*}{$\begin{array}{l}\text { Drug } \\
\text { score }\end{array}$} \\
\hline & Mutagenicity & Tumorigenicity & Irritancy & $\begin{array}{c}\text { Reproductive } \\
\text { effects }\end{array}$ & & & \\
\hline 1 & & & & & -4.80 & 2.13 & 0.53 \\
\hline 2 & & & & & -3.91 & 3.11 & 0.59 \\
\hline 3 & & & & & -4.62 & 3.54 & 0.59 \\
\hline 4 & & & & & -4.25 & 2.86 & 0.68 \\
\hline 5 & & & & & -4.98 & 5.35 & 0.54 \\
\hline 6 & & & & & -3.75 & 1.91 & 0.73 \\
\hline 7 & & & & & -4.29 & 3.05 & 0.69 \\
\hline 8 & & & & & -1.92 & 5.22 & 0.82 \\
\hline 9 & & & & & -4.95 & 5.01 & 0.53 \\
\hline 10 & & & & & -4.24 & 4.23 & 0.66 \\
\hline 11 & & & & & -3.56 & 4.35 & 0.79 \\
\hline 12 & & & & & -3.48 & 5.62 & 0.78 \\
\hline 13 & & & & & -4.67 & 5.82 & 0.58 \\
\hline 14 & & & & & -3.78 & 6.14 & 0.74 \\
\hline 15 & & & & & -5.27 & 3.02 & 0.47 \\
\hline 16 & & & & . & -2.05 & 3.88 & 0.79 \\
\hline 17 & & & & & -3.77 & 5.10 & 0.74 \\
\hline 18 & & & & & -5.39 & 1.42 & 0.43 \\
\hline 19 & & & & & -5.28 & 6.94 & 0.49 \\
\hline 20 & & & & & -3.12 & 6.25 & 0.78 \\
\hline Dexamethasone & & & & & -3.25 & 3.17 & 0.79 \\
\hline Hydrocortisone & & & & & -3.18 & 3.31 & 0.49 \\
\hline
\end{tabular}




\section{Molecular properties and drug-likeness}

In the framework of our study, the conformity of the compounds to the Lipinski rule of five was evaluated [14] this simple rule is based on the observation that most biologically active drugs have a molecular weight of approximately 500 or less, $\log P$ values not exceeding 5 , hydrogen donor sites of not more than 5 , and hydrogen bond acceptor sites not greater than 10 . In addition, the polar topological surface (TPSA) and the number of rotary links have been linked to the bioavailability of drugs [15]. Molecular properties (TPSA, nrotb, miLogP, OH-NH interaction, $\mathrm{ON}$ interaction, molecular weight and number of violations of Lipinski's rule) of the compounds were calculated using molinspiration software and compared to the values of standard drugs: dexamethasone and the natural hormone (Hydrocortisone). Drug distribution results from tissue diffusion which itself depends on drug size and lipophilicity, therefore prediction of permeability across gastrointestinal epithelial cells, the blood-brain barrier and the brain, which can help interpret pharmacokinetic results and understand the behavior of these products in the body [17]. On the other hand, highly lipophilic drugs will only be absorbed after dissolving in the digestive tract. This is confirmed by lipinski, the value of miLogP must be estimated between 0.4 and +5 [18]. The polar topological surface (TPSA) is a very useful parameter for predicting the drug transport properties. It gives information on the polarity of molecules. TPSA is calculated on the basis of the methodology published by Ertl et al. [19]. It is defined as the sum of the surfaces of the polar atoms usually, oxygen, nitrogen, sulfur, chlorine, and hydrogen in a molecule. Thus, it is closely linked to the hydrogen bonding potential of a compound. TPSA has been shown to be a very good descriptor characterizing drug absorption, including intestinal absorption, bioavailability, and penetration of the blood-brain barrier. Molecules with TPSA values of around $140 \AA^{2}$ or more should exhibit poor intestinal absorption [20]. Considering these recommendations, the results presented in the table indicate that all the compounds analyzed have TPSA values. $<140 \AA^{2}$. So, they should have good intestinal absorption.

The number of rotatable bonds is used to identify the flexibility of the molecule. it has been shown that to be a drug with very good oral bioavailability, and for a chemical structure to be similar to drugs, according to Lipinski's rule, the number of rotatable bonds must be less than or equal to 5. Among all the compounds tested, 2.6 and 7 were rigid because they have no rotary bond. All the compounds tested have rotatable bonds $<5$ and they may not have problems of bioavailability according to the results cited in (Table 2.). Furthermore, all the compounds fulfill the Lipinski rule [21], which implies a good oral bioavailability.

Table 2: Physicochemical property of the compounds (1-20) and standard drugs.

\begin{tabular}{|c|c|c|c|c|c|c|c|c|}
\hline \multirow[b]{2}{*}{ Compd. $\mathrm{N}^{\circ}$} & \multicolumn{8}{|c|}{ Molecular properties } \\
\hline & Volume & TPSA $^{\text {a }}$ & n-ROTB b & HBA ${ }^{c}$ & HBD d & $\operatorname{miLogP} \mathrm{e}$ & $\mathbf{M W}^{\mathrm{f}}$ & $\begin{array}{l}\text { Lipinski's } \\
\text { violations }\end{array}$ \\
\hline Rule & - & - & - & $<10$ & $<5$ & $\leq 5$ & $<500$ & $\leq 1$ \\
\hline 1 & 426.12 & 87.74 & 4 & 5 & 2 & 4.11 & 492.74 & 0 \\
\hline 2 & 345.13 & 63.60 & 0 & 4 & 1 & 2.50 & 392.44 & 0 \\
\hline 3 & 416.97 & 96.97 & 4 & 6 & 2 & 3.57 & 476.52 & 0 \\
\hline 4 & 361.48 & 74.60 & 3 & 4 & 2 & 3.06 & 430.49 & 0 \\
\hline 5 & 429.53 & 86.62 & 4 & 5 & 3 & 3.29 & 491.60 & 0 \\
\hline 6 & 328.55 & 63.60 & 0 & 4 & 1 & 2.02 & 378.42 & 0 \\
\hline 7 & 361.72 & 63.60 & 0 & 4 & 1 & 2.86 & 406.47 & 0 \\
\hline 8 & 393.68 & 99.76 & 3 & 6 & 3 & 1.92 & 425.52 & 0 \\
\hline 9 & 426.12 & 83.83 & 4 & 5 & 2 & 4.21 & 492.58 & 0 \\
\hline 10 & 388.68 & 74.60 & 3 & 4 & 2 & 3.39 & 456.53 & 0 \\
\hline 11 & 349.50 & 100.62 & 1 & 5 & 4 & 1.58 & 395.45 & 0 \\
\hline 12 & 367.17 & 86.92 & 1 & 5 & 3 & 1.96 & 409.47 & 0 \\
\hline 13 & 461.75 & 95.86 & 5 & 6 & 3 & 3.18 & 499.67 & 0 \\
\hline 14 & 383.97 & 86.62 & 2 & 5 & 3 & 2.33 & 423.50 & 0 \\
\hline 15 & 442.25 & 74.60 & 4 & 4 & 2 & 4.97 & 486.70 & 0 \\
\hline 16 & 398.29 & 99.76 & 3 & 6 & 3 & 1.91 & 443.51 & 0 \\
\hline 17 & 376.16 & 95.86 & 2 & 6 & 3 & 1.92 & 425.47 & 0 \\
\hline 18 & 441.35 & 87.74 & 4 & 5 & 2 & 3.58 & 488.57 & 0 \\
\hline 19 & 453.12 & 86.62 & 4 & 5 & 3 & 3.99 & 483.67 & 0 \\
\hline 20 & 384.11 & 77.84 & 1 & 5 & 2 & 1.76 & 423.50 & 0 \\
\hline Dexamethasone & 358.07 & 94.83 & 2 & 5 & 3 & 2.06 & 392.47 & 0 \\
\hline Hydrocortisone & 343.36 & 94.83 & 2 & 5 & 3 & 1.62 & 362.47 & 0 \\
\hline
\end{tabular}

a Topological polar surface area;

b number of rotatable bonds;

${ }^{c}$ number of hydrogen bond acceptors;

d number of hydrogen bond donors;

e logarithm of partition coefficient between n-octanol and water;

f MW. molecular weight. 


\section{Bioactivity score}

The bioactivity scores of the compounds for the drug targets have also been predicted by Molinspirations and are presented in the Table 3. A molecule with a bioactivity scores greater than 0.00 is likely to exhibit considerable biological activity, while values between -0.50 and 0.00 are moderately active and if the score is less than - 0.50 , it is presumed to be inactive [22].

Corticosteroids have the originality of exerting their actions through essentially genomic effects where all the compounds presented in the Table 3 . indicate positive values. These results suggest that the new compounds have shown promising properties, in terms of binding to the nuclear receptor in order to exercise transcriptional activity there. In addition, the data were compared to standard references, indicating that 11 compounds have a better bioactivity score on the nuclear receptor than the reference standard, more precisely dexamethasone (see Table 3.) This makes the hypothesis that these elements may be more effective therapeutically possible.

GCs also induce rapid effects occurring within minutes or even seconds of their application. In fact, inhibiting the activation of protein $G$ abolishes the rapid effects of glucocorticoids. The results presented in the table. Indicate that the compounds 5,13,15 and 19 have values between 0.18 to 0.04 of GPCR ligand; so, they should have a moderate genomic effect. On the other hand, the non-genomic actions of the other compounds presented in the Table 3. have a better score than the reference therefore these structures could also be important from a clinical point of view, because the GC induce short-term therapeutic effects in patients with asthma, rheumatoid arthritis, and allergic rhinitis [23].

Protein kinases appear to be essential protagonists in the regulation of cell signaling pathways. Indeed, these enzymes play a fundamental role in cell growth and differentiation as well as in apoptosis phenomena. The inhibition of the latter causes many undesirable effects. Unfortunately, 6 compounds analyzed can cause these effects moderately (Table 3.).

The results clearly reveal that the compounds analyzed, and dexamethasone could inhibit proteases and other enzymes.

Table 3: Bioactivity score of the compounds according to Molinspirations Cheminformatics.

\begin{tabular}{|c|c|c|c|c|c|c|}
\hline \multirow{2}{*}{ Compd. $\mathrm{N}^{\circ}$} & \multicolumn{6}{|c|}{ Drug-likeness } \\
\hline & GPCRL & ICM & KI & NRL & PI & EI \\
\hline 1 & 0.07 & -0.26 & -0.78 & 1.30 & 0.67 & 0.62 \\
\hline 2 & 0.18 & 0.07 & -0.54 & 1.73 & 0.94 & 0.78 \\
\hline 3 & 0.04 & -0.15 & -0.59 & 1.35 & 0.59 & 0.59 \\
\hline 4 & 0.22 & 0.06 & -0.55 & 2.06 & 1.03 & 0.95 \\
\hline 5 & 0.00 & -0.20 & -0.50 & 1.19 & 0.62 & 0.54 \\
\hline 6 & 0.24 & 0.11 & -0.42 & 1.79 & 0.93 & 0.83 \\
\hline 7 & 0.16 & -0.01 & -0.54 & 1.66 & 0.94 & 0.74 \\
\hline 8 & 0.16 & -0.22 & -0.42 & 1.01 & 0.20 & 0.66 \\
\hline 9 & 0.07 & -0.08 & -0.52 & 1.45 & 0.68 & 0.64 \\
\hline 10 & 0.14 & -0.01 & -0.71 & 1.80 & 0.94 & 0.86 \\
\hline 11 & 0.23 & 0.03 & -0.43 & 1.78 & 1.03 & 0.82 \\
\hline 12 & 0.27 & -0.03 & -0.50 & 1.70 & 1.06 & 0.80 \\
\hline 13 & -0.15 & -0.37 & -0.58 & 0.56 & 0.01 & 0.33 \\
\hline 14 & 0.25 & -0.06 & -0.52 & 1.62 & 0.98 & 0.76 \\
\hline 15 & -0.11 & -0.39 & -0.82 & 0.81 & 0.14 & 0.46 \\
\hline 16 & 0.17 & -0.18 & -0.45 & 1.32 & 0.60 & 0.76 \\
\hline 17 & 0.23 & -0.09 & -0.36 & 1.60 & 1.13 & 0.88 \\
\hline 18 & 0.13 & -0.15 & -0.63 & 1.39 & 0.66 & 0.61 \\
\hline 19 & -0.18 & -0.45 & -0.66 & 0.62 & -0.03 & 0.34 \\
\hline 20 & 0.23 & -0.01 & -0.43 & 1.67 & 0.94 & 0.72 \\
\hline Dexamethasone & 0.03 & -0.21 & -0.81 & 1.59 & 0.76 & 0.78 \\
\hline Hydrocortisone & -0.00 & -0.29 & -0.85 & 1.17 & 0.09 & 0.63 \\
\hline
\end{tabular}

GPCRL: GPCR ligand, ICM: ion channel modulator, KI: kinase inhibitor, NRL: nuclear receptor ligand, PI: protease inhibitor, EI: enzyme inhibitor. 


\section{ADMET prediction for the proposed compounds}

One of the essential qualities of a drug is its ability to cross the intestinal membrane (absorption) to then reach the systemic circulation: this is called the bioavailability of a drug. Among the many transporters present in the intestinal membrane, we find the P-gp which is part of the ABC transporters. It is present in the apical membrane of epithelial cells of the gastrointestinal tract. It plays a protective role by preventing the passage of potentially dangerous molecules from the intestine into the blood. Many drugs are found to be substrates for this transporter (Colchicine, azithromycin, losartan, lopinavir, dexamethasone, etc.), which limits their bioavailability and reduces the treatment efficiency [24]. The results presented in the table indicate that all the compounds are substrates of P-gp, but they remain very permeable to the intestinal membrane with the exception of compound 15. On the other hand, Drug metabolism is a crucial step in the detoxification and elimination of drugs by chemical conversion to more hydrophilic molecules whose elimination by body fluids is facilitated. Only a few CYPs are strongly involved in the transformation and elimination of active ingredients in humans. These are CYP 1A2, 2A6, 2B6, 2C9 / 19, 2D6, 2E1 and 3A4. Inhibition of CYP and other enzymes in drug metabolism can affect the clearance and consequently the serum level of these drugs. The therapeutic effectiveness of a drug or its active form can also be varied. Therefore, the concomitant oral administration of a CYP3A4 inhibitor and a drug that is a substrate for the same isoenzyme can lead to a very large increase in the plasma concentration of the drug and thus lead to increased toxicity. ABC

Exploring these parameters may offer new strategies that could bridge the gap between early stage drug discovery, preclinical and clinical trials, as well as gain insight into their reaction in the body, allowing the chemist Medicinal to introduce new functional groups on the molecule to dodge the metabolic pathways capable of giving very polar compounds which can be eliminated very easily from the body. As a result, it can help synthesize metabolically stable drugs, as well as avoid drug interactions. For this purpose, the compounds were submitted to learn the potential of compounds having inhibitor of CYP with human hepatic microsomes CYP (Table 4.). Only the compounds 6,7,8,11 and 16 are not inhibitors of all CYP.

Table 4: Pharmacokinetics of compounds.

\begin{tabular}{|c|c|c|c|c|c|c|c|c|}
\hline Compd. $\mathrm{N}^{\circ}$ & $\begin{array}{c}\text { GI } \\
\text { absorptiona }^{a}\end{array}$ & $\begin{array}{c}\text { BBB } \\
\text { permeant }\end{array}$ & $\begin{array}{c}\text { P-gp } \\
\text { substratec }\end{array}$ & $\begin{array}{l}\text { CYP1A2 } \\
\text { inhibitor }\end{array}$ & $\begin{array}{l}\text { CYP2C19 } \\
\text { inhibitor }\end{array}$ & $\begin{array}{l}\text { CYP2C9 } \\
\text { inhibitor }\end{array}$ & $\begin{array}{l}\text { CYP2D6 } \\
\text { inhibitor }\end{array}$ & $\begin{array}{c}\text { CYP3A4 } \\
\text { inhibitor }\end{array}$ \\
\hline 1 & High & No & Yes & No & No & No & No & Yes \\
\hline 2 & High & Yes & Yes & No & No & No & No & No \\
\hline 3 & High & No & Yes & No & No & No & No & Yes \\
\hline 4 & High & No & Yes & No & No & No & No & Yes \\
\hline 5 & High & No & Yes & No & No & No & No & Yes \\
\hline 6 & High & Yes & Yes & No & No & No & No & No \\
\hline 7 & High & Yes & Yes & No & No & No & No & No \\
\hline 8 & High & No & Yes & No & No & No & No & No \\
\hline 9 & High & No & Yes & No & No & No & No & Yes \\
\hline 10 & High & No & Yes & No & No & No & No & Yes \\
\hline 11 & High & No & Yes & No & No & No & No & No \\
\hline 12 & High & No & Yes & No & No & No & No & Yes \\
\hline 13 & High & No & Yes & No & No & No & No & Yes \\
\hline 14 & High & No & Yes & No & No & No & No & Yes \\
\hline 15 & Low & No & Yes & No & No & Yes & No & No \\
\hline 16 & High & No & Yes & No & No & No & No & No \\
\hline 17 & High & No & Yes & No & No & No & No & Yes \\
\hline 18 & High & No & Yes & No & No & No & No & Yes \\
\hline 19 & High & No & Yes & No & No & Yes & No & Yes \\
\hline 20 & High & Yes & Yes & No & No & No & No & Yes \\
\hline Dexamethasone & High & No & Yes & No & No & No & No & No \\
\hline Hydrocortisone & High & No & Yes & No & No & No & No & No \\
\hline
\end{tabular}

a: Gastrointestinal absorption; b: Blood brain barrier; c: Permeability-Glycoprotein; CYP: Cytochrome P450; Log $\boldsymbol{K}_{\mathbf{p} \text { : skin }}$ permeation. 


\section{Molecular docking analysis}

Molecular docking is used to predict the binding mode of ligands within the binding site of target proteins [25]. Protein enhancement and grid generation are required steps for suitable docking. A protein, PDB ID: 1M2Z was retrieved in PDB format from Protein data bank from which the threedimensional X-ray crystallographic structure of human glucocorticoid receptor (PDB ID: 1M2Z) was taken. Protein preparation Wizard of Maestro version 11.1 was used for refining the structure while the removal of water and optimization of $\mathrm{H}$-bond was done. Minimization of heavy atom molecule at RMSD $(0.30 \AA)$ by using force filed OPLS2005 were used to release the process of minimization. Protein was prepared by eliminating water molecules, needless atoms, removing the alternate conformations and adding hydrogen.

The grid generation describes a region in a receptor where binding interaction can happen. Before launching the molecular docking, the geometry of all molecules needs to be optimized (Vora et al., 2019). There are basically four steps involved in the docking scores which include HTVS (highthroughput virtual screening), SP (standard precision), XP (extra precision) docking, and followed by MM-GBSA based binding free energy calculation for top-scored ligand-protein complexes.

The docking scores results of the investigated molecules are illustrated in Table 5.; the scores are arranged in descending order from smallest the negative value to the majority negative value. It was found that all molecules have poses in the receptor site, and two molecules with better scores when compared to the score of the marketed molecule (Hydrocortisone). Furthermore, Analysis of molecular docking-based interaction profiles of the selected compounds including Dexamethasone and Hydrocortisone revealed that they have formed hydrogen bond (H-bond) interactions with several amino acid residues of (1M2Z) , particularly residues Asn-564, Arg-611, Gln-642 and Gln570.

Three-dimensional docking model of Dexamethasone, Hydrocortisone, compound (4) and compound (11) with the target protein (PDB ID: 1M2Z) are portrayed in Fig 3. This figure showed that all compounds have common amino acids. We also notice that Dexamethasone and Hydrocortisone created two hydrogen bonds with Asn-564.

Table 5: Docking results of 20 novel ligands and standard ligands with the target protein $\quad$ (PDB ID: 1M2Z)

\begin{tabular}{|c|c|c|c|c|c|}
\hline Ligand name & $\begin{array}{l}\text { Docking } \\
\text { Score }\end{array}$ & $\begin{array}{l}\text { Glide } \\
\text { energy }\end{array}$ & $\begin{array}{l}\text { Glide ligand } \\
\text { efficiency }\end{array}$ & $\mathrm{N}^{\circ}$ of $\mathrm{H}$ bonds & Amino acids \\
\hline 1 & -10.715 & -24.666 & -0.315 & 2 & Asn564, Arg611 \\
\hline 2 & -12.157 & -62.966 & -0.433 & 2 & Gln570, Asn564 \\
\hline 3 & -11.914 & -31.967 & -0.350 & 1 & Asn564 \\
\hline 4 & -13.391 & -52.892 & -0.462 & 3 & Asn564, Arg611, Gln642 \\
\hline 5 & -12.223 & -21.673 & -0.360 & 3 & Asn564*, Arg611 \\
\hline 6 & -12.612 & -59.571 & -0.467 & 2 & Asn564, Arg611 \\
\hline 7 & -11.171 & -54.315 & -0.385 & 2 & Gln570, Arg611 \\
\hline 8 & -11.088 & -37.664 & -0.358 & 3 & Gln570, Asn564, Gln642 \\
\hline 9 & -11.707 & -26.112 & -0.334 & 2 & Asn564, Leu732 \\
\hline 10 & -12.407 & -61.132 & -0.400 & 1 & Asn564 \\
\hline 11 & -14.654 & -70.767 & -0.532 & 5 & Gln570, Asn564, Thr739, Gln642* \\
\hline 12 & -13.119 & -61.249 & -0.452 & 3 & Gln570, Asn564, Gln642 \\
\hline 13 & -10.371 & -20.076 & -0.296 & 3 & Gln570, Asn564, Arg611 \\
\hline 14 & -13.188 & -57.461 & -0.440 & 4 & Gln570, Asn564, Gln642* \\
\hline 15 & -11.050 & -19.098 & -0.335 & 4 & Asn564*, Arg611, Met560 \\
\hline 16 & -11.620 & -36.280 & -0.363 & 3 & Gln570, Asn564, Leu732 \\
\hline 17 & -13.060 & -54.344 & -0.435 & 4 & Asn564*, Arg611, Gln642 \\
\hline 18 & -12.676 & -48.375 & -0.362 & 4 & Met560, Asn564*, Arg611 \\
\hline 19 & -10.403 & -24.029 & -0.306 & 2 & Asn564* \\
\hline 20 & -12.195 & -47.126 & -0.407 & 2 & Asn564, Gln642 \\
\hline Dexamethasone & -14.209 & -56.936 & -0.507 & 5 & Gln570, Asn564*, Arg611, Gln642 \\
\hline Hydrocortisone & -13.668 & -60.356 & -0.526 & 4 & Asn564*, Arg611, Gln642 \\
\hline
\end{tabular}

*(Two hydrogen bonding interactions with the same amino acid) 


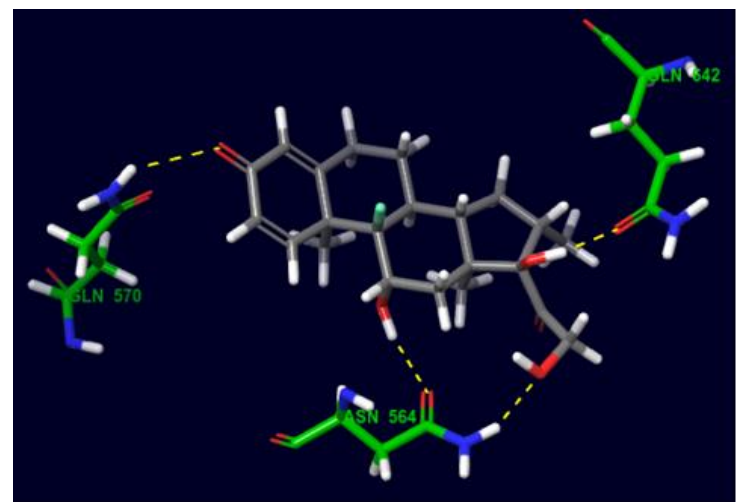

a

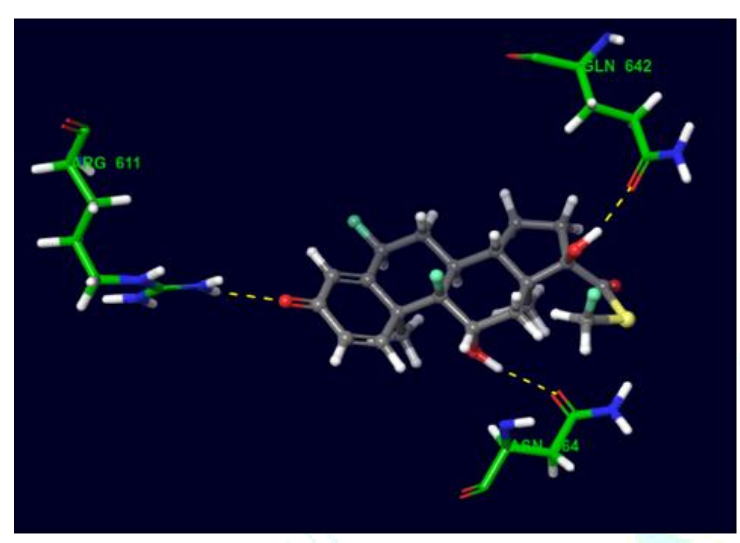

c

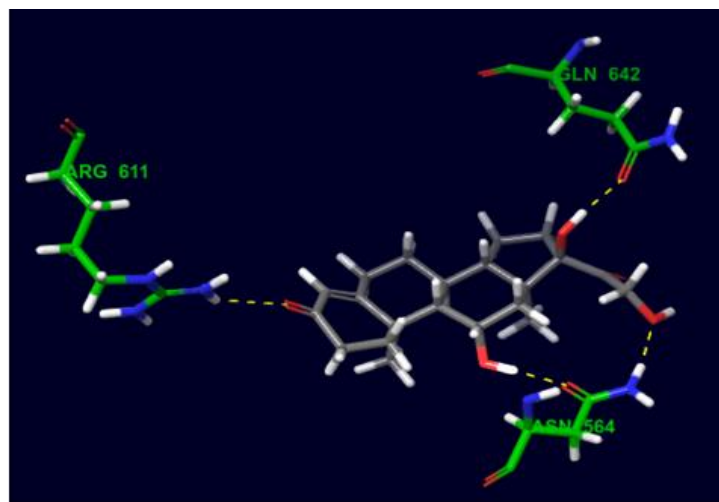

b

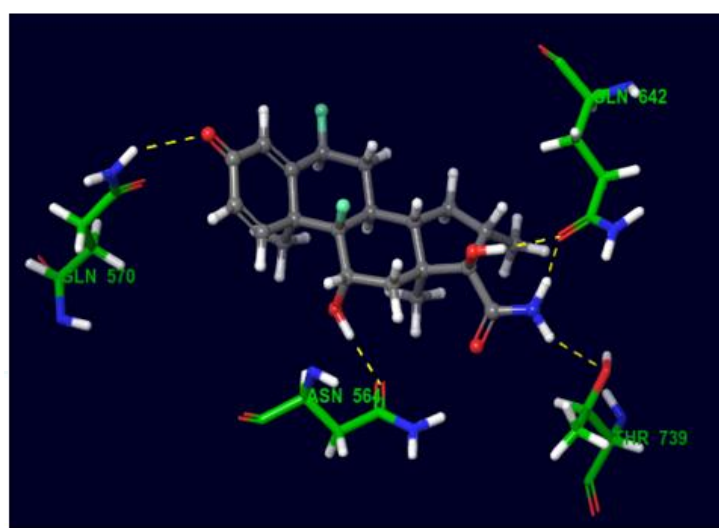

d

Figure 2: Three-dimensional docking model of a) Dexamethasone, b) Hydrocortisone, c) compound (4) and d) compound (11) with the target protein (PDB ID: 1M2Z)

\section{CONCLUSION}

In this study, Molinspirtion and Osiris guided better understanding of the relationship between the physicochemical properties and biological activity observed for the 20 chosen compounds. Oral bioavailability, molecular descriptors, drug likeness properties and bioactivity were estimated as per the Lipinski rules of five and results outcomes of all compound showed good oral bioavailability. Drug relevant properties were predicted, and results indicates that all compounds get good drug score. All the synthetic compounds were found to have a high degree of lipophilicity, which designates a good lipid solubility that will help drug to interact with the membranes. Compound 11 exhibited higher drug score, bioactivity score and revealed good drug relevant properties. Furthermore, drug likeness analysis suggests that the designed drug derivatives require serious transformation in order to obtain appropriate oral absorption and brain penetration for potential therapeutic applications.

Comparing the docking values, drug-likeliness, ADME profile and toxicity analysis of the derivatives to the parent compound Hydrocortisone, the derivatives are found to have favorable scores thus suggesting that the problem of poor bioavailability, pharmacokinetics and solubility can be overcome by structural changes, and serve as promising lead candidates for alternative anti-asthma therapy. We should mention that Bioinformatics analyses and molecular docking gives only suggestion or prediction, these data may do not reflect real biological properties. So, bioavailability, pharmacokinetic or toxicity should be verified on animals with special authorization.
This article is distributed under the terms of the Creative Commons Attribution- NonCommercial 4.0 International License (http://creativecommons.org/licenses/by-hc/4.0/), which permits any noncommercial use, distribution, and reproduction in any medium, provided you give appropriate credit to the original author(s) and the source, provide a link to the Creative Commons license, and indicate if changes were made

\section{REFERENCES}

[1] Katsaounou P, Buhl R, Brusselle G, Pfister P, Martínez R, Wahn $\mathrm{U}$, Bousquet J, Omalizumab as alternative to chronic use of oral corticosteroids in severe asthma, Respir. Med. 2019; 150:51-62.

[2] Choo XN, Pavord ID, Morbidity associated with oral corticosteroids in patients with severe asthma, Thorax. 2016; 71:302-304.

[3] Bourdin A, Molinari N, Vachier I, Pahus L, Suehs C, Chanez P, Mortality: a neglected outcome in OCS-treated severe asthma, Eur. Respir. J. 2017; 50.

[4] Ray A, Raundhal M, Oriss TB, Ray P, Wenzel SE, Current concepts of severe asthma, J. Clin. Invest. 2016; 126.

[5] Humbert T, Taillé C, Mala L, Gros VL, Just J, Molimard M, Omalizumab effectiveness in patients with severe allergic asthma according to blood eosinophil count: the STELLAIR study, Eur. Respir. J. 2018; 51.

[6] Guevara JP, Ducharme FM, Keren R, Nihtianova S, Zorc J, Cochrane review: Inhaled corticosteroids versus sodium cromoglycate in children and adults with asthma, Evid. -Based Child Health Cochrane Rev. J. 2006; 1:1025-1095.

[7] Carroll TL, Chronic Cough, Plural Publishing, 2019. 
[8] Durham AL, Caramori G, Chung KF, Adcock IM, Targeted antiinflammatory therapeutics in asthma and chronic obstructive lung disease, Transl. Res. 2016; 167

[9] Rahman I, Gilmour PS, Jimenez LA, MacNee W, Oxidative stress and TNF-a induce histone Acetylation and NF-кВ/AP-1 activation in Alveolar epithelial cells: Potential mechanism In gene transcription in lung inflammation, in: V. Vallyathan, X. Shi, V. Castranova (Eds.), Oxyg. Radic. Cell Inj. Dis., Springer US, Boston, MA, 2002: pp. 239-248.

[10] Zhang J, Yang Y, Liu W, Schlenk D, Liu J, Glucocorticoid and mineralocorticoid receptors and corticosteroid homeostasis are potential targets for endocrine-disrupting chemicals, 2019.

[11] Daley-Yates PT, Inhaled corticosteroids: potency, dose equivalence and therapeutic index, Br. J. Clin. Pharmacol. 80 (2015) 372-380.

[12] Matera MG, Rinaldi B, Calzetta L, Rogliani P, Cazzola M, Pharmacokinetics and pharmacodynamics of inhaled corticosteroids for asthma treatment, Pulm. Pharmacol. Ther. 2019; 101828.

[13] Bououden W, Benguerba Y, Computational Quantum Chemical Study, Drug-Likeness and In Silico Cytotoxicity Evaluation of Some Steroidal Anti-Inflammatory Drugs, J. Drug Deliv. Ther 2020; 10(3-s):68-74

[14] Parvez A, Meshram J, Tiwari V, Sheik J, Dongre R, Youssoufi MH, Ben Hadda T, Pharmacophores modeling in terms of prediction of theoretical physico-chemical properties and verification by experimental correlations of novel coumarin derivatives produced via Betti's protocol, Eur. J. Med. Chem. 2010; 45:43704378.

[15] Tian S, J. er al, The application of in silico drug-likeness predictions in pharmaceutical research, Adv. Drug Deliv. Rev. $2015 ; 86: 2-10$.
[16] Rashid M, Design, synthesis and ADMET prediction of bisbenzimidazole as anticancer agent, Bioorganic Chem. 2020; 96:103576.

[17] Passeleu-Le Bourdonnec C, Carrupt PA, Scherrmann JM, Martel $\mathrm{S}$, Methodologies to assess drug permeation through the bloodbrain barrier for pharmaceutical research, Pharm. Res. 2013; 30:2729-2756.

[18] Estevez Yet al, Trypanocidal properties, structure-activity relationship and computational studies of quinoxaline 1, 4-di-Noxide derivatives, Exp. Parasitol. 2011; 127:745-751.

[19] Ertl P, Rohde B, Selzer P, Fast calculation of molecular polar surface area as a sum of fragment-based contributions and its application to the prediction of drug transport properties, J. Med. Chem. 2000; 43:3714-3717.

[20] Ertl P, Polar surface area, Mol. Drug Prop. 2008; 111-126.

[21] Zhang MQ, Wilkinson B, Drug discovery beyond the 'rule-offive,' Curr. Opin. Biotechnol. 2007; 18:478-488.

[22] Paramashivam SK, Elayaperumal K, Bhagavan Natarajan B, Devi Ramamoorthy M, Balasubramanian S, Dhiraviam KN, In silico pharmacokinetic and molecular docking studies of small molecules derived from Indigofera aspalathoides Vahl targeting receptor tyrosine kinases, Bioinformation. 2015; 11:73.

[23] Löwenberg M, Stahn C, Hommes DW, Buttgereit F, Novel insights into mechanisms of glucocorticoid action and the development of new glucocorticoid receptor ligands, Steroids. 2008; 73:1025-1029.

[24] Batista da Silva Junior J, Marinho Dezani T, Bersani Dezani A, Helena dos Reis Serra C, evaluating potential P-gp substrates: Main aspects to choose the adequate permeability model for assessing gastrointestinal drug absorption, Mini Rev. Med. Chem. 2015; 15:858-871.

[25] Dar AM, Mir S, Molecular docking: approaches, types, applications and basic challenges, J Anal Bioanal Tech. 2017; 8:1-3. 